\title{
NEOPLASIA E DISPNEIA
}

\section{NEOPLASIA AND DYSPNEA}

Marcos Vieira Rangel Pereira1, Marcos Marcelo Neves Filho², Evandro Tito Oliveira2, Daniele Micima Moribe ${ }^{2}$

Caso Clínico: Homem de 74 anos, hipertenso, adenocarcinoma de próstata confirmado por biópsia em 2014 e condrossarcoma de fêmur direito confirmado por biópsia em 2009 após início de dor intensa na articulação coxo-femoral associado a tumoração local com aumento progressivo. Neoplasia metastática pulmonar confirmada logo após diagnóstico de condrossarcoma. Relatava dispneia aos grandes esforços há aproximadamente 6 anos, com piora lenta e progressiva, se tornando aos moderados esforços no último ano. Quinze dias antes do internamento apresentou dispneia aos mínimos esforços e em repouso, com piora abrupta dos sintomas. Apresentava tosse crônica, sem alteração do padrão no período. Negava dor, febre, hemoptóico ou outras queixas. Ao exame físico, apresentava-se taquidispneico com esforço respiratório e uso de musculaturas acessórias evidentes, agitado e desconfortável no leito. Murmúrio vesicular reduzido em base direita e com crepitantes em terço médio e base esquerda, além de sibilos inspiratórios difusos. Evoluiu a óbito cinco dias após a admissão hospitalar. Antes do óbito, uma angiotomografia de tórax havia sido realizada. Qual o diagnóstico?
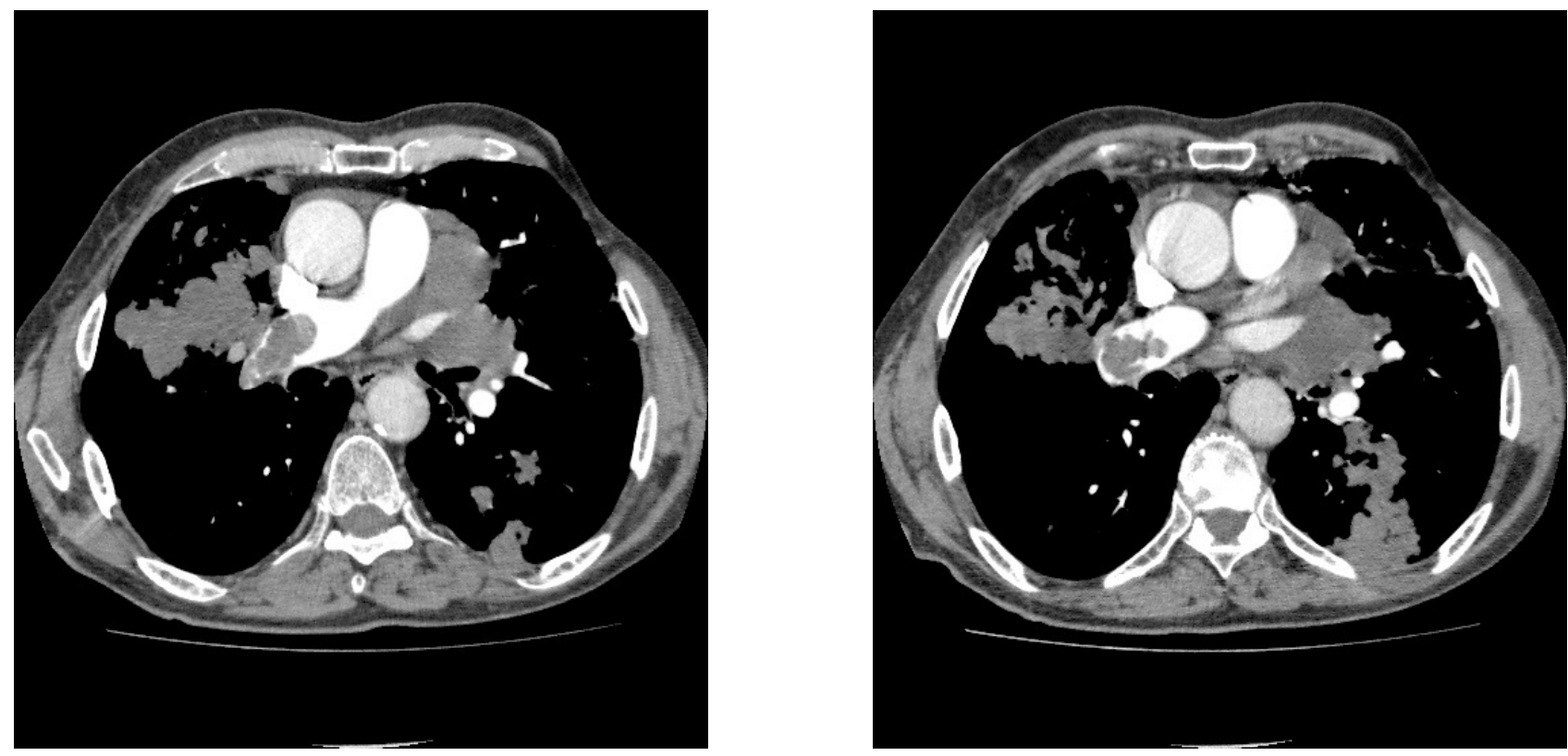

A. Embolia pulmonar decorrente de trombose venosa profunda.

B. Pneumonia.

C. Embolia gordurosa.

D. Trombo tumoral.

E. Evolução da doença pulmonar metastática.

1. Hospital de Clínicas da Universidade Federal do Paraná, Curitiba, Brasil

2. Universidade Federal do Paraná, Curitiba, Brasil

Contato do Autor / Mail to:

Marcos Vieira Rangel Pereira

Rua General Carneiro, 181, 110 andar 


\section{RESPOSTA D - Trombo tumoral}

A embolia tumoral ocorre quando material celular proveniente de um tumor é encontrado nas artérias pulmonares, causando, assim, obstrução mecânica e progressiva. Trata-se de um evento muito raro e de difícil diagnóstico, cujo risco de ocorrência é aumentado após a realização de procedimentos que potencialmente promovam a fragmentação e a liberação de massa tumoral na circulação, tais como: quimioterapia, radioterapia e ressecção cirúrgica1,2.

Morfologicamente, a embolia pulmonar pode ser dividida em macroembolia e microembolia, sendo a última a apresentação mais freqüente ${ }^{1}$. Os êmbolos tumorais ocorrem frequentemente de maneira subclínica, sendo um acho de autópsia em até $26 \%$ dos pacientes com algum tumor sólido maligno ${ }^{3}$. Os principais sítios primários, correspondendo a cerca de $75 \%$ dos casos, são mama (28\%), pulmão (21\%), estômago (12\%) e próstata (12\%). Macroêmbolos são descritos principalmente em carcinomas renais e endometriais, bem como no adenocarcinoma gastrointestinal ${ }^{1}$.

O curso clínico é subagudo, com dispnéia progressiva que evolui num intervalo de dias a meses, associada a taquicardia, taquipnéia, dor torácica do tipo pleurítica, hemoptise e perda de peso. Em presença de insuficiência respiratória hipoxêmica associada a acidose láctica e um rápido declínio do estado geral de um paciente gravemente doente, sem achados significativos em radiografia de tórax, a hipótese de tromboembolismo tumoral deve ser aventada2,3. Os êmbolos pulmonares devem ainda ser diferenciados de linfangite carcinomatosa e de trombos embólicos decorrentes de coagulação intravascular disseminada secundária a um processo tumoral, o que pode se mostrar desafiador 3 .

\section{ACHADOS DE IMAGEM}

$\mathrm{Na}$ microembolia, como o material tumoral emboligênico não é visto na TC, o diagnóstico repousa essencialmente nos achados parenquimatosos correspondentes a sinais indiretos de obstrução como edema pulmonar, infarto, empiema ou hipertensão pulmonar. Quando as células tumorais preenchem as artérias centrolobulares, desencadeiam uma extensa reação de hiperplasia intimal das pequenas artérias pulmonares, o que gera o sinal da "árvore em brotamento" que é, no entanto, inespecífico, pois pode ser encontrado em outras doenças intersticiais pulmonares. Em suma, a microembolia pode mimetizar os achados de imagem de pneumonia, tuberculose e doença pulmonar intersticial ${ }^{1}$.

Já na macroembolia, os achados de imagem são indiferenciáveis do TEP. Como fator confusional adicional, os pacientes portadores de neoplasias malignas possuem um risco adicional de desenvolver tal condição4. Como os êmbolos tumorais são resistentes à terapia fibrinolítica, tendem a causar obstrução progressiva e irreversível. A embolia tumoral pode ainda apresentar-se como invasão microvascular pulmonar dentro de um quadro de envolvimento linfático generalizado, levando à formação de infiltrados intersticiais difusos $1,2,4$.

O diagnóstico radiológico é extremamente difícil. Até mesmo a angiotomografia, considerada o padrão ouro na detecção de eventos tromboembólicos, possui uma limitada sensibilidade e especificidade nesses casos. 0 único procedimento que pode demonstrar de maneira inequívoca a etiologia da hipoxemia nesses pacientes é a biópsia pulmonar, na qual evidenciam-se células tumorais na vasculatura pulmonar ${ }^{3}$.

\section{TRATAMENTO E PROGNÓSTICO}

Apesar da embolia tumoral normalmente não ser grande o suficiente para causar morte súbita, o prognóstico é reservado. Portanto, evita-se a realização de procedimentos invasivos como terapêutica. Apenas a remoção cirúrgica de macroêmbolos pode ter alguma utilidade na evolução clínica. 0 essencial, nesse caso, é tratar o tumor primário1,4.

\section{REFERÊNCIAS}

1. Bach AG, Restrepo CS, Abbas J, Villanueva A, Lorenzo Dus MJ, Schöpf R, et al. Imaging of nonthrombotic pulmonary embolism: Biological materials, nonbiological materials, and foreign bodies. Eur J Radiol [Internet]. Elsevier Ireland Ltd; 2013;82(3):e120-41. Available from: http://dx.doi.org/10.1016/j.ejrad.2012.09.019

2. Roberts KE, Hamele-Bena D, Saqi A, Stein CA, Cole RP. Pulmonary tumor embolism: A review of the literature. Am J Med. 2003;115(3):228-32.

3. Deeren D, Verbeken E, Vanderschueren S, Wilmer A, Bobbaers H, Meersseman W. Cancer Presenting As Fatal Pulmonary Tumour Embolism. Acta Clin Belg [Internet]. 2006;61(1):30-4. Available from: http://www.maneyonline.com/doi/abs/10.1179/acb. 2006.006

4. Pena E, Dennie C, Franquet T, Milroy C. Nonthrombotic Pulmonary Embolism: A Radiological Perspective. Semin Ultrasound, CT MRI [Internet]. Elsevier Inc.; 2012;33(6):522-34. Available from: http://dx.doi.org/10.1053/j.sult.2012.05.002 